PROCEEDINGS OF THE

AMERICAN MATHEMATICAL SOCIETY

Volume 133, Number 7, Pages 2157-2164

S 0002-9939(05)07907-4

Article electronically published on February 24, 2005

\title{
A TOPOLOGICAL PALEY-WIENER PROPERTY FOR LOCALLY COMPACT GROUPS
}

\author{
EBERHARD KANIUTH, ANTHONY T. LAU, AND GÜNTER SCHLICHTING
}

(Communicated by Andreas Seeger)

\begin{abstract}
We investigate a certain topological Paley-Wiener property and show, for instance, that compact-free nilpotent groups and simply connected solvable groups share this property.
\end{abstract}

\section{INTRODUCTION}

Let $f$ be a bounded and compactly supported measurable function on $\mathbb{R}^{n}(f \in$ $\left.L_{c}^{\infty}\left(\mathbb{R}^{n}\right)\right)$. By the classical Paley-Wiener theorem, the Fourier transform $\widehat{f}$ of $f$ extends to an entire function on $\mathbb{C}^{n}$. It follows that $f=0$ whenever $\widehat{f}$ vanishes on a set of positive Lebesgue measure. If, more generally, $G$ is a (second countable) unimodular locally compact group of type I and $\widehat{G}$ denotes the dual space of $G$, then $G$ is said to satisfy the weak Paley-Wiener property if the operator-valued Fourier transform $\pi \rightarrow \pi(f)$ of a non-zero function $f \in L_{c}^{\infty}(G)$ cannot vanish on a set of positive Plancherel measure on $\widehat{G}$. This weak Paley-Wiener property has been established by several authors for simply connected nilpotent Lie groups [1], 7], 14, [15] and later for completely solvable Lie groups [8].

In this paper we introduce and study another Paley-Wiener type property which can be defined for arbitrary locally compact groups $G$. We say that $G$ has the topological Paley-Wiener property if for every non-zero $f \in L_{c}^{\infty}(G)$, the map $\pi \rightarrow$ $\pi(f)$ cannot vanish on any non-empty open subset of the reduced dual $\widehat{G}_{r}$ of $G$. Our main result is an extension theorem which asserts that if $G$ contains a closed normal subgroup $N$ that has the topological Paley-Wiener property, then so does $G$, provided that $G / N$ is abelian and compact-free (Theorem 2.2). As a consequence, compact-free nilpotent locally compact groups and simply connected solvable Lie groups share this topological Paley-Wiener property (Corollaries 2.4 and 2.5).

\section{Preliminaries And some Basic Results}

Let $G$ be a locally compact group with fixed left Haar measure, and let $C^{*}(G)$ be the completion of the convolution algebra $L^{1}(G)$ with respect to the norm $\|f\|_{C^{*}(G)}=\sup \{\|\pi(f)\|\}$, where the supremum is taken over all $*$-representations $\pi$ of $L^{1}(G)$ as an algebra of bounded linear operators in a Hilbert space. Let $P(G)$ be the set of all continuous positive definite functions on $G$, and let $B(G)$ denote the

Received by the editors December 2, 2003.

2000 Mathematics Subject Classification. Primary 22D10, 22E25, 43A30, 43A40.

The second author was supported by NSERC grant A7679. 
linear span of $P(G)$. Then $B(G)$ can be identified with the dual space $C^{*}(G)^{*}$ of $C^{*}(G)$ by the pairing $\langle f, u\rangle=\int_{G} f(x) u(x) d x$ for $f \in L^{1}(G)$ und $u \in B(G)$. With pointwise multiplication and the dual norm, $B(G)$ is a commutative Banach algebra, called the Fourier-Stieltjes algebra of $G$. The Fourier algebra $A(G)$ is the norm closure in $B(G)$ of $A_{c}(G)=B(G) \cap C_{c}(G)$, the compactly supported functions in $B(G)$. For details regarding $B(G)$ and $A(G)$, see the fundamental paper [5].

As is customary, we shall use the same letter, for example $\pi$, for a unitary representation of $G$ and the corresponding *-representations of $L^{1}(G)$ and $C^{*}(G)$. For any representation $\pi$ of $G$, let $A_{\pi}(G)$ denote the closed linear subspace of $B(G)$ generated by all coefficient functions of $\pi$, and $B_{\pi}(G)$ the $w^{*}$-closure of $A_{\pi}(G)$ in $B(G)$. Then $B_{\pi}(G)$ can be identified with the dual of the $C^{*}$-algebra $\pi\left(C^{*}(G)\right)$. Note that, when $\rho=\rho_{G}$ denotes the regular representation of $G$ on $L^{2}(G)$, then $A_{\rho}(G)=A(G)$, and $B_{\rho}(G)$ equals $B(G)$ if and only if $G$ is amenable.

Moreover, we need to recall some definitions from representation theory. If $S$ and $T$ are sets of unitary representations, then $S$ is weakly contained in $T(S \prec T)$ if $\bigcap\{\operatorname{ker} \sigma: \sigma \in S\} \supseteq \bigcap\{\operatorname{ker} \tau: \tau \in T\}$, where $\operatorname{ker} \pi$ denotes the $C^{*}$-kernel of a representation $\pi$. The sets $S$ and $T$ are said to be weakly equivalent $(S \sim T)$ if $S \prec T$ and $T \prec S$. Then, for any two representations $\sigma$ and $\tau, \sigma \prec \tau$ if and only $B_{\sigma}(G) \subseteq B_{\tau}(G)$. The support of a representation $\pi, \operatorname{supp} \pi$, is the closed subset of $\widehat{G}$ consisting of all $\tau \in \widehat{G}$ such that $\tau \prec \pi$. Thus $\pi \sim \widehat{G}$ if and only if $\operatorname{supp} \pi=\widehat{G}$. As general references to dual spaces of locally compact groups and weak containment properties, we mention [4] and 6].

Lemma 1.1. If $G$ contains a non-trivial compact normal subgroup $K$, then $G$ cannot have the topological Paley-Wiener property.

Proof. Let $\mu_{K}$ denote the (normalized) Haar measure of $K$. Let $\pi$ be an irreducible representation of $G$ such that $\pi \notin \widehat{G / K}$. If $\sigma$ is an irreducible subrepresentation of $\left.\pi\right|_{K}$, then, since $\sigma \neq 1_{K}$, by the orthogonality relations $\int_{K}\langle\sigma(k) \xi, \eta\rangle d k=0$ for all $\xi, \eta \in \mathcal{H}_{\sigma}$. It follows that $\pi\left(\mu_{K}\right)=0$. Now, consider $f=g * \mu_{K}, g \in C_{c}(G)$. Then $f \in L_{c}^{\infty}(G)$ and $\pi(f)=0$ for all $\pi \in \widehat{G}_{r} \backslash \widehat{G / K}$, a non-empty open subset of $\widehat{G}_{r}$.

For any $f \in L^{1}(G)$, let

$$
Z_{f}=\left\{\pi \in \widehat{G}_{r}: \pi(f)=0\right\},
$$

the zero set of $f$ in $\widehat{G}_{r}$. Note that when studying the topological Paley-Wiener property, we can always assume that the function $f \in L_{c}^{\infty}(G)$ actually belongs to $A_{c}(G)$ since $f * f^{*}$ is positive definite and $Z_{f * f^{*}}=Z_{f}$. We say that $f \in A_{c}(G)$ generates $B_{\rho}(G)$ in the $w^{*}$-topology if the linear span of all two-sided translates of $f$ is $w^{*}$-dense in $B_{\rho}(G)$.

Lemma 1.2. Let $G$ be a locally compact group and let $f \in A_{c}(G)$. Then $Z_{f}$ has empty interior if and only if $f$ generates $B_{\rho}(G)$ in the $w^{*}$-topology.

Proof. Let $I_{f}$ denote the closed ideal of $C_{r}^{*}(G)$ generated by $\rho(f)$. If $\stackrel{\circ}{Z}_{f} \neq \emptyset$, then the non-zero ideal $J$ of $C_{r}^{*}(G)$ defined by $\widehat{J}=\stackrel{\circ}{Z}_{f}$ satisfies $J \cap I_{f}=\{0\}$ since $\widehat{I_{f}}=\widehat{G}_{r} \backslash Z_{f}$. Conversely, if there exists a non-zero ideal $J$ of $C_{r}^{*}(G)$ such that $J \cap I_{f}=\{0\}$, then $\widehat{J} \cap\left(\widehat{G}_{r} \backslash Z_{f}\right)=\widehat{J} \cap \widehat{I_{f}}=\left(J \cap I_{f}\right)^{\wedge}=\emptyset$ and hence $\emptyset \neq \widehat{J} \subseteq Z_{f}$, so 
$\stackrel{\circ}{Z}_{f} \neq \emptyset$. Therefore, to prove the lemma it suffices to show that there exists a nonzero ideal $J$ of $C_{r}^{*}(G)$ with $J \cap I_{f}=\{0\}$ if and only if $E_{f}$, the $w^{*}$-closed subspace of $B_{\rho}(G)$ generated by all two-sided translates of $f$, does not coincide with $B_{\rho}(G)$.

Let $\Delta$ denote the modular function of $G$ and for any function $h$ on $G$, define $\widetilde{h}$ by $\widetilde{h}(y)=\Delta\left(y^{-1}\right) h\left(y^{-1}\right)$. Then note that $\langle\rho(h) \rho(g), u\rangle=\langle\rho(g), \widetilde{h} * u\rangle$ for all $g \in C_{c}(G)$ and $h, u \in A_{c}(G)$, and hence $\langle\rho(h) T, u\rangle=\langle T, \widetilde{h} * u\rangle$ for all $T \in C_{r}^{*}(G)$ and $h, u \in A_{c}(G)$.

Now let $J$ be an ideal as above. Then $\langle T, \widetilde{f} * u\rangle=0$ for all $T \in J$ and $u \in A_{c}(G)$ since $\rho(f) T=0$. Writing $f$ as $f(\cdot)=\langle\pi(\cdot) \xi, \eta\rangle$ for some representation $\pi$ of $G$ and $\xi, \eta \in \mathcal{H}_{\pi}$ and choosing $u$ such that $u \geq 0$ and $\|u\|_{1}=1$, we have

$$
\begin{aligned}
(f * u)(x)-f(x) & =\int_{G} u\left(y^{-1}\right)\langle\pi(x) \pi(y) \xi, \eta\rangle d y-\langle\pi(x) \xi, \eta\rangle \\
& =\left\langle\pi(x)\left(\int_{G} u\left(y^{-1}\right)(\pi(y) \xi-\xi) d y\right), \eta\right\rangle .
\end{aligned}
$$

This implies that

$$
\|f * u-f\|_{B_{\rho}(G)} \leq\|\eta\| \sup \left\{\|\pi(y) \xi-\xi\|: y^{-1} \in \operatorname{supp} u\right\} .
$$

Since $\langle T, f * u\rangle=0$, taking for $u \in A_{c}(G)$ the usual approximate identity for $L^{1}(G)$, it follows that

$$
|\langle T, f\rangle| \leq\|T\| \cdot\|f * u-f\|_{B_{\rho}(G)} \rightarrow 0
$$

for each $T \in J$. Since $J^{\perp}$ is a $w^{*}$-closed two-sided translation invariant subspace of $B_{\rho}(G)$, we conclude that $E_{f} \subseteq J^{\perp}$, a proper subspace of $B_{\rho}(G)$.

Conversely, suppose that $E_{f} \neq B_{\rho}(G)$ and let

$$
J=\left\{T \in C_{r}^{*}(G):\langle T, u\rangle=0 \text { for all } u \in E_{f}\right\} .
$$

Then $J$ is a non-zero closed ideal $C_{r}^{*}(G)$. We show that $J \cap I_{f}=\{0\}$. For $T \in$ $C_{r}^{*}(G), s, t \in G$ and $u \in A_{c}(G)$, we have

$$
\left\langle\rho\left(L_{t^{-1}} R_{s^{-1}} \tilde{f}\right) T, u\right\rangle=\Delta(t s)\left\langle T,\left(L_{s} R_{t} f\right) * u\right\rangle .
$$

Therefore, it will follow that $J \cap I_{f}=\{0\}$ once we have verified that if $E$ is a $w^{*}$ closed translation invariant subspace of $B_{\rho}(G)$, then $E * u \subseteq E$ for every $u \in A_{c}(G)$. This can be seen as follows. If $v(\cdot)=\langle\pi(\cdot) \xi, \eta\rangle \in E$, then

$$
(v * u)(\cdot)=\left\langle\pi(\cdot)\left(\int_{G} u\left(y^{-1}\right) \pi(y) \xi d y\right), \eta\right\rangle .
$$

Given $\epsilon>0$, there exist $y_{1}, \ldots, y_{n} \in G$ and $c_{1}, \ldots, c_{n} \geq 0$ such that

$$
\left\|\int_{G} u\left(y^{-1}\right) \pi(y) \xi d y-\sum_{j=1}^{n} c_{j} \pi\left(y_{j}\right) \xi\right\| \leq \epsilon .
$$

Then $w(\cdot)=\sum_{j=1}^{n} c_{j}\left\langle\pi(\cdot) \pi\left(y_{j}\right) \xi, \eta\right\rangle \in E$ and

$$
\|v * u-w\|_{B_{\rho}(G)} \leq\|\eta\| \cdot\left\|\int_{G} u\left(y^{-1}\right) \pi(y) \xi d y-\sum_{j=1}^{n} c_{j} \pi\left(y_{j}\right) \xi\right\| \leq \epsilon\|\eta\| .
$$

This finishes the proof. 
It is worth pointing out that, according to the following lemma, the topological Paley-Wiener property is equivalent to a dichotomy for the subspaces $\overline{B_{\pi}(G) \cap A_{c}(G)}$ of $A(G)$, which has been investigated in Section 5 of $[13$.

Lemma 1.3. For any locally compact group $G$, the following two conditions are equivalent:

(i) G has the topological Paley-Wiener property.

(ii) For any unitary representation $\pi$ of $G$, either $B_{\pi}(G) \cap A_{c}(G)=\{0\}$ or $\overline{B_{\pi}(G) \cap A_{c}(G)}=A(G)$.

Proof. (i) $\Rightarrow$ (ii) Let $\pi$ be any representation of $G$, and suppose there exists $f \in$ $B_{\pi}(G) \cap A_{c}(G), f \neq 0$. By (i), $Z_{f}$ has empty interior and hence $f$ generates $B_{\rho}(G)$ in the $w^{*}$-topology (Lemma 1.2). Thus

$$
B_{\rho}(G) \subseteq{\overline{B_{\pi}(G) \cap A_{c}(G)}}^{w^{*}} \subseteq B_{\pi}(G),
$$

and this in turn implies that

$$
A(G)=\overline{B_{\rho}(G) \cap A_{c}(G)} \subseteq \overline{B_{\pi}(G) \cap A_{c}(G)},
$$

as required.

(ii) $\Rightarrow$ (i) Let $f$ be a non-zero function in $A_{c}(G)$, and let $E$ denote the closed two-sided invariant subspace of $A(G)$ generated by $f$. There exists a representation $\pi$ of $G$ such that $E=A_{\pi}(G)$ [2] Théorème 3.17]. By hypothesis, either $B_{\pi}(G) \cap$ $A_{c}(G)=\{0\}$ or $\overline{B_{\pi}(G) \cap A_{c}(G)}=A(G)$. However, $f \in B_{\pi}(G) \cap A_{c}(G)$. Therefore $\overline{B_{\pi}(G) \cap A_{c}(G)}=A(G)$ and hence

$$
\bar{E}^{w^{*}} \subseteq B_{\rho}(G)=\overline{A(G)}^{w^{*}}={\overline{B_{\pi}(G) \cap A_{c}(G)}}^{w^{*}} \subseteq \bar{E}^{w^{*}},
$$

so that $f$ generates $B_{\rho}(G)$ in the $w^{*}$-topology. By Lemma $1.2, \stackrel{\circ}{Z}_{f} \neq \emptyset$.

An interesting consequence of Lemma 1.3 is that the topological Paley-Wiener property can be interpreted as an approximation property for $A(G)$.

A locally compact group $G$ is called a SIN-group (a group with small invariant neighbourhoods) if there exists a neighbourhood basis of the identity consisting of sets $V$ such that $x^{-1} V x=V$ for all $x \in G$. SIN-groups provide a large class of locally compact groups comprising all abelian groups, compact groups and discrete groups. For the structure theory of SIN-groups, see [11.

Theorem 1.4. For a SIN-group $G$, the following two conditions are equivalent:

(i) $G$ contains no non-trivial compact normal subgroup.

(ii) $G$ has the topological Paley-Wiener property.

Proof. This is an immediate consequence of Lemma 1.3 and Theorem 5.6 of [13].

We conclude this section with the observation that the topological Paley-Wiener property is in general not inherited by normal subgroups. Indeed, an easy example showing this can be constructed as follows. Let $F$ be a finite abelian group and let $N=\sum_{j=1}^{\infty} F_{j}$ be the direct sum of copies $F_{j}$ of $F$. Let $G=S_{\infty} \ltimes N$ be the semidirect product, where the infinite symmetric group $S_{\infty}$ acts on $N$ by permuting the index set $\mathbb{N}$. Obviously, $G$ is a discrete ICC group and hence has the weak Paley-Wiener property (Theorem 1.4), whereas the normal subgroup $N$ is an abelian torsiongroup. 


\section{EXTENSIONS AND APPLICATIONS}

In this section we are going to prove two theorems of the nature that if a locally compact group $G$ contains a closed normal subgroup that has the topological Paley-Wiener property, then under certain additional hypotheses, $G$ also has the topological Paley-Wiener property. Both of these results apply to various (classes of) locally compact groups. A similar, but much less applicable, result has been shown in 13, Proposition 5.3].

Let $H$ be a closed subgroup of $G$ and let $\tau$ be a representation of $H$. The representation of $G$ induced by $\tau$ is denoted $\operatorname{ind}_{H}^{G} \tau$. In the sequel we shall use that $\pi \otimes \operatorname{ind}_{H}^{G} \tau=\operatorname{ind}_{H}^{G}\left(\left.\pi\right|_{H} \otimes \tau\right)$ and that $\pi \prec \operatorname{ind}_{H}^{G}\left(\left.\pi\right|_{H}\right)$ when $G$ is amenable [10. Theorem 5.1].

Lemma 2.1. Let $H$ be a closed subgroup of the locally compact group $G$. Let $U$ be a non-empty open subset of $\widehat{G}_{r}$, and let

$$
V=\left\{\tau \in \widehat{H}_{r}: \operatorname{supp}\left(\operatorname{ind}_{H}^{G} \tau\right) \nsubseteq \widehat{G}_{r} \backslash U\right\} .
$$

Then $V$ is non-empty and open in $\widehat{H}_{r}$.

Proof. To show that $\widehat{H}_{r} \backslash V$ is closed in $\widehat{H}_{r}$, let $\left(\tau_{\alpha}\right)_{\alpha}$ be a net in $\widehat{H}_{r} \backslash V$ such that $\tau_{\alpha} \rightarrow \tau$ for some $\tau \in \widehat{H}_{r}$. Since inducing is continuous in Fell's subgroup representation topology [6, Theorem 4.2], $\operatorname{ind}_{H}^{G} \tau_{\alpha} \rightarrow \operatorname{ind}_{H}^{G} \tau$. Since $\operatorname{ind}_{H}^{G} \tau_{\alpha} \prec$ $\widehat{G}_{r} \backslash U$ for all $\alpha$ and $\widehat{G}_{r} \backslash U$ is closed in $\widehat{G}_{r}$, it follows that $\operatorname{supp}\left(\operatorname{ind}_{H}^{G} \tau\right) \subseteq \widehat{G}_{r} \backslash U$ and hence $\tau \in \widehat{H}_{r} \backslash V$.

Assume that $V=\emptyset$, that is, $\operatorname{supp}\left(\operatorname{ind}_{H}^{G} \tau\right) \subseteq \widehat{G}_{r} \backslash U$ for all $\tau \in \widehat{H}_{r}$. Then, since $\rho_{G}=\operatorname{ind}_{H}^{G} \rho_{H}$, we obtain that

$$
\widehat{G}_{r} \sim\left\{\operatorname{ind}_{H}^{G} \tau: \tau \in \widehat{H}_{r}\right\} \sim \bigcup_{\tau \in \widehat{H}_{r}} \operatorname{supp}\left(\operatorname{ind}_{H}^{G} \tau\right) \subseteq \widehat{G}_{r} \backslash U .
$$

This contradiction shows that $V$ is non-empty.

Recall that a locally compact group $G$ is said to be compact-free if the identity is the only element of $G$ generating a relatively compact subgroup.

Theorem 2.2. Let $G$ be a locally compact group containing a closed normal subgroup $N$ such that $G / N$ is abelian and compact-free. If $N$ has the topological PaleyWiener property, then so does $G$.

Proof. Let $f \in C_{c}(G)$ be such that $\stackrel{\circ}{Z}_{f} \neq \emptyset$. We have to show that $\left.L_{x} f\right|_{N}=0$ for all $x \in G$. Temporarily, fix $\pi \in \stackrel{\circ}{f}_{f}$ and $\xi, \eta \in \mathcal{H}_{\pi}$, and consider on $\widehat{G / N}$ the function

$$
\chi \rightarrow\langle(\pi \otimes \chi)(f)(\xi \otimes 1), \eta \otimes 1\rangle .
$$

Define $h \in C_{c}(G / N)$ by

$$
h(x N)=\int_{N} f(x n)\langle\pi(x n) \xi, \eta\rangle d n,
$$

$x \in G$. Then, for $\chi \in \widehat{G / N}$,

$$
\begin{aligned}
\widehat{h}(\chi) & =\int_{G / N} \chi(x N)\left(\int_{N} f(x n)\langle\pi(x n) \xi, \eta\rangle\right) d(x N) \\
& =\langle(\pi \otimes \chi)(f)(\xi \otimes 1), \eta \otimes 1\rangle .
\end{aligned}
$$


Since $G / N$ is abelian and compact-free, $\widehat{h}$ can vanish only on a set of Haar measure zero in $\widehat{G / N}$ [13, Lemma 5.1]. However, $\widehat{h}$ vanishes on the set

$$
X=\left\{\chi \in \widehat{G / N}: \pi \otimes \chi \in \stackrel{\circ}{Z}_{f}\right\}
$$

and $X$ is a non-empty open subset of $\widehat{G / N}$ since $\pi \in X$ and the mapping $\chi \rightarrow \pi \otimes \chi$ from $\widehat{G / N}$ into $\widehat{G}$ is continuous. We conclude that $h=0$. Since $\xi, \eta \in \mathcal{H}_{\pi}$ are arbitrary, it follows that $(\pi \otimes \chi)(f)=0$ for all $\pi \in \stackrel{\circ}{f}_{f}$ and $\chi \in \widehat{G / N}$. Now,

$$
\pi \otimes \widehat{G / N}=\{\pi \otimes \chi: \chi \in \widehat{G / N}\} \sim \pi \otimes \operatorname{ind}_{N}^{G} 1_{N}=\operatorname{ind}_{N}^{G}\left(\left.\pi\right|_{N}\right),
$$

and since $\pi \prec \rho_{G}$ implies that $\left.\pi\right|_{N} \prec \rho_{N}$, we get that

$$
\operatorname{ind}_{N}^{G}\left(\left.\pi\right|_{N}\right) \prec \operatorname{ind}_{N}^{G} \rho_{N}=\rho_{G}
$$

and therefore $\pi \otimes \widehat{G / N} \subseteq \widehat{G}_{r}$. Thus we have seen that $\stackrel{\circ}{f}_{f} \otimes \widehat{G / N} \subseteq Z_{f}$. Since $\sigma \rightarrow \sigma \otimes \chi$ is a homeomorphism of $\widehat{G}_{r}$,

$$
\stackrel{\circ}{Z}_{f} \otimes \widehat{G / N}=\bigcup_{\chi \in \widehat{G / N}} \stackrel{\circ}{Z}_{f} \otimes\{\chi\}
$$

is open in $\widehat{G}_{r}$, whence $\stackrel{\circ}{Z}_{f} \otimes \widehat{G / N}=\stackrel{\circ}{Z}_{f}$.

Now, let $x \in G$ and define $g \in C_{c}(N)$ by $g(n)=f(x n)$. We have to show that $\stackrel{\circ}{Z}_{g} \neq \emptyset$ for all such functions $g$. To that end, define a subset $V$ of $\widehat{N}_{r}$ by

$$
V=\left\{\tau \in \widehat{N}_{r}: \operatorname{supp}\left(\operatorname{ind}_{N}^{G} \tau\right) \not \widehat{G}_{r} \backslash \stackrel{\circ}{Z}_{f}\right\} .
$$

By Lemma 2.1, $V$ is non-empty and open in $\widehat{N}_{r}$. It remains to verify that $V \subseteq Z_{g}$. Fix $\tau \in V$ and choose a net $\left(\pi_{\alpha}\right)_{\alpha}$ in $\widehat{G}_{r}$ such that $\left.\pi_{\alpha}\right|_{N} \rightarrow \tau$ in Fell's topology. We claim that $\pi_{\alpha} \in \stackrel{\circ}{Z}_{f}$ eventually. Towards a contradiction, after passing to a subnet if necessary, let us assume that $\pi_{\alpha} \notin \stackrel{\circ}{Z}_{f}$ for all $\alpha$. Since $\stackrel{\circ}{f}_{f}=\stackrel{\circ}{Z}_{f} \otimes \widehat{G / N}$, it follows that

$$
\operatorname{ind}\left(\left.\pi_{\alpha}\right|_{N}\right) \sim \pi_{\alpha} \otimes \widehat{G / N} \subseteq \widehat{G}_{r} \backslash \stackrel{\circ}{f}_{f}
$$

for all $\alpha$. Therefore, for each $\sigma \in \operatorname{supp}\left(\left.\pi_{\alpha}\right|_{N}\right)$,

$$
\operatorname{supp}\left(\operatorname{ind}_{N}^{G} \sigma\right) \subseteq \operatorname{supp}\left(\operatorname{ind}_{N}^{G}\left(\left.\pi_{\alpha}\right|_{N}\right)\right) \subseteq \widehat{G}_{r} \backslash \stackrel{\circ}{Z}_{f},
$$

and hence $\sigma \in \widehat{N}_{r} \backslash V$. Thus $\left.\pi_{\alpha}\right|_{N} \prec \widehat{N}_{r} \backslash V$ and so $\tau \in \widehat{N}_{r} \backslash V$. This contradiction shows that $\pi_{\alpha} \in \stackrel{\circ}{Z}_{f}$ eventually. Since

$$
\operatorname{ind}_{N}^{G}\left(\left.\pi_{\alpha}\right|_{N}\right) \sim \pi_{\alpha} \otimes \widehat{G / N} \subseteq \stackrel{\circ}{Z}_{f} \otimes \widehat{G / N} \subseteq Z_{f}
$$

and $\operatorname{ind}\left(\left.\pi_{\alpha}\right|_{N}\right) \rightarrow \operatorname{ind}_{N}^{G} \tau$, it follows that $\operatorname{supp}\left(\operatorname{ind}_{N}^{G} \tau\right) \subseteq Z_{f} . \operatorname{But}_{\operatorname{ind}_{N}^{G}}^{G} \tau(f)=0$ implies that $\tau\left(\left.L_{x} f\right|_{N}\right)=0$ (compare [16, Lemma 2.1]), that is, $\tau \in Z_{g}$. This finishes the proof.

Corollary 2.3. Suppose that $G$ possesses a sequence $H_{0}=\{e\} \subseteq H_{1} \subseteq \ldots \subseteq H_{m}=$ $G$ of closed subgroups such that $H_{j-1}$ is normal in $H_{j}$ and $H_{j} / \bar{H}_{j-1}$ is compact-free abelian $(1 \leq j \leq m)$. Then $G$ has the topological Paley-Wiener property.

Proof. Using Theorem 2.2, the statement follows immediately by induction on $m$. 
Corollary 2.4. Let $G$ be a nilpotent locally compact group. Then $G$ has the topological Paley-Wiener property if and only if $G$ is compact-free.

Proof. Suppose that $G$ has the topological Paley-Wiener property. Then, by Lemma 1.1, $G$ cannot have a non-trivial compact normal subgroup. It is easy to see that this implies that $G^{c}=\{e\}$. Indeed, let $\{e\}=Z_{0}(G) \subseteq Z_{1}(G) \subseteq \ldots$ denote the ascending central series of $G$ and, assuming that $G^{c} \neq\{e\}$, let $m \geq 0$ be maximal with the property that $G^{c} \cap Z_{m}(G)=\{e\}$. Then pick $a \in G^{c} \cap Z_{m+1}(G), a \neq e$, and let $H$ be the closed subgroup of $G$ generated by $Z_{m}(G)$ and $a$. Then $H$ is normal in $G$ and hence $H^{c}$ is a closed normal subgroup of $G$. However, $H^{c}$ is the closed subgroup generated by $a$, which is compact.

Conversely, if $G$ is compact-free, then $G_{0}$, the connected component of the identity, is simply connected and $D=G / G_{0}$ is discrete and torsion-free [9] Theorem 8.3]. We now apply Corollary 2.3 as follows. If $G=G_{0}$, then $Z_{m}(G)$ and the quotient group $G / Z_{m}(G)$ are simply connected for all $m$. Then induction yields that $G$ has the topological Paley-Wiener property. In the general case, note that $D / Z_{m}(D)$ is torsion-free for all $m[3$, Corollary 2.11]. Thus, a further induction on the length of nilpotency of $D$ shows that $G$ has the topological Paley-Wiener property.

Corollary 2.5. Let $G$ be a simply connected solvable Lie group. Then $G$ has the topological Paley-Wiener property.

Proof. By the structure theory of simply connected solvable Lie groups, there exists a sequence $H_{0}=\{e\} \subseteq H_{1} \subseteq \ldots \subseteq H_{m}=G$ of closed subgroups such that $H_{j-1}$ is normal in $H_{j}$ and $H_{j} / H_{j-1}=\mathbb{R}$ (see [12, Satz III.3.30]). Thus $G$ has the topological Paley-Wiener property by Corollary 2.3.

Theorem 2.6. Let $G$ be a locally compact group and let $N$ be a closed normal subgroup of $G$ such that $G / N$ is amenable. Suppose there exists a dense subset $T$ of $\widehat{N}_{r}$ such that for each $\tau \in T$, $\operatorname{ind}_{N}^{G} \tau$ is weakly equivalent to some irreducible representation. If $N$ has the topological Paley-Wiener property, then so does $G$.

Proof. Let $f \in C_{c}(G)$ such that $\stackrel{\circ}{Z}_{f} \neq \emptyset$. By Lemma 2.1, the set

$$
V=\left\{\tau \in \widehat{N}_{r}: \operatorname{supp}\left(\operatorname{ind}_{N}^{G} \tau\right) \nsubseteq \widehat{G}_{r} \backslash \stackrel{\circ}{f}_{f}\right\}
$$

is non-empty and open in $\widehat{N}_{r}$. Since $T$ is dense in $\widehat{N}_{r}, T \cap V$ is dense in $V$. By hypothesis, for every $\tau \in T$ there exists $\pi_{\tau} \in \widehat{G}$ such that $\pi_{\tau} \sim \operatorname{ind}_{N}^{G} \tau$. Since $G / N$ is amenable, $\pi_{\tau} \in \widehat{G}_{r}$. Then $\pi_{\tau} \in \check{Z}_{f}$ for each $\tau \in T \cap V$. Indeed, for such $\tau$,

$$
\pi_{\tau} \sim \operatorname{supp}\left(\operatorname{ind}_{N}^{G} \tau\right) \not \subset \widehat{G}_{r} \backslash \stackrel{\circ}{Z}_{f}
$$

and hence $\pi_{\tau} \in \stackrel{\circ}{Z}_{f}$. Now, for any $\tau \in V$ there exists a net $\left(\tau_{\alpha}\right)_{\alpha}$ in $T \cap V$ converging to $\tau$. Then

$$
\pi_{\tau_{\alpha}} \sim \operatorname{ind}_{N}^{G} \tau_{\alpha} \rightarrow \operatorname{ind}_{N}^{G} \tau
$$

and since $\pi_{\tau_{\alpha}}(f)=0$ for all $\alpha$, it follows that $\operatorname{ind}_{N}^{G} \tau(f)=0$. This in turn implies that $\tau\left(\left.L_{x} f\right|_{N}\right)=0$ for all $x \in G$. Since $N$ has the topological Paley-Wiener property, we conclude that $f=0$.

We finish the paper with an example to which Theorem 2.6 applies, but neither Theorem 2.2 nor Proposition 5.3 of [13] applies. 
Example 2.7. Let $\mathbb{Z}_{2}=\{1,-1\}, K=\prod_{n=1}^{\infty} \mathbb{Z}_{2}$ and $N=\sum_{n=1}^{\infty} \mathbb{Z}$, the restricted direct sum of copies of $\mathbb{Z}$. Let $G$ be the semidirect product $G=K \ltimes N$, where $K$ acts on $N$ by $(\epsilon \cdot x)_{n}=\epsilon_{n} x_{n}\left(\epsilon=\left(\epsilon_{n}\right)_{n} \in K, x=\left(x_{n}\right)_{n} \in N\right)$. Identifying $\widehat{\mathbb{Z}}$ with $\mathbb{T}$, we have $\widehat{N}=\prod_{n=1}^{\infty} \mathbb{T}$ and, for $z=\left(z_{n}\right)_{n} \in \widehat{N}$ and $\epsilon=\left(\epsilon_{n}\right)_{n} \in K, \epsilon \cdot z=z$ if and only if $z_{n}=\overline{z_{n}}$ for all $n \in \mathbb{N}$ such that $\epsilon_{n}=-1$. Now, $T=\bigcap_{n=1}^{\infty}\left\{z \in \widehat{N}: \overline{z_{n}} \neq z_{n}\right\}$ is dense in $\widehat{N}$, and each $z \in T$ has a trivial stability group in $K$ and hence the associated induced representation of $G$ is irreducible. By Theorem 2.6, $G$ has the topological Paley-Wiener property.

In [13. Example 5.4] we have given several other examples of locally compact groups having the topological Paley-Wiener property.

\section{REFERENCES}

[1] D. Arnal and J. Ludwig, Q.U.P. and and Paley-Wiener properties of unimodular, especially nilpotent, Lie groups, Proc. Amer. Math. Soc. 125 (1997), 1071-1080. MR1353372 (97f:43004)

[2] G. Arsac, Sur l'espace de Banach engendré par les coefficients d'une représentation unitaire, Publ. Dép. Math. (Lyon) 13 (1976), 1-101. MR0444833 (56:3180)

[3] G. Baumslag, Lectures on nilpotent groups, Amer. Math. Soc. Regional Conference Series No. 2 (1971). MR0283082 (44:315)

[4] J. Dixmier, $C^{*}$-algebras, North-Holland, 1977. MR0458185|(56:16388)|

[5] P. Eymard, L'algèbre de Fourier d'un groupe localement compact, Bull. Soc. Math. France 92 (1964), 181-236. MR0228628 (37:4208)

[6] J.M.G. Fell, Weak containment and induced representations. II, Trans. Amer. Math. Soc. 110 (1964), 424-447. MR0159898 (28:3114)

[7] G. Garimella, Un théorème de Paley-Wiener pour les groupes de Lie nilpotents, J. Lie Theory 5 (1995), 165-172. MR.1389426 (97f:22016)

[8] G. Garimella, Weak Paley-Wiener property for completely solvable Lie groups, Pacific J. Math. 187 (1999), 51-63. MF 1674293 (2000a:22011)

[9] V.M. Gluskov, Locally nilpotent locally bicompact groups, Trudy Moscov. Obshch. 4 (1955), 291-332. (Russian) MR0072422 (17:281b)

[10] F.F. Greenleaf, Amenable actions of locally compact groups, J. Funct. Anal. 4 (1969), 295315. MR0246999 (40:268)

[11] S. Grosser and M. Moskowitz, Compactness conditions in topological groups, J. reine angew. Math. 246 (1971), 1-40. MR0284541 (44:1766)

[12] J. Hilgert and K.-H. Neeb, Lie-Gruppen und Lie-Algebren, Vieweg, 1991.

[13] E. Kaniuth, A.T. Lau and G. Schlichting, Lebesgue type decomposition of subspaces of Fourier-Stieltjes algebras, Trans. Amer. Math. Soc. 355 (2003), 1467-1490. MR1946400 (2004c:43004)

[14] V. Kisil, A theorem of Paley-Wiener type for nilpotent Lie groups, Ukrainian Math. J. 50 (1998), 1786-1788. MR1706645 (2000m:22010)

[15] R.L. Lipsman and J. Rosenberg, The behavior of Fourier transforms for nilpotent Lie groups, Trans. Amer. Math. Soc. 348 (1996), 1031-1050. MR1370646 (97d:22008)

[16] J. Ludwig, Good ideals in the group algebra of a nilpotent Lie group, Math. Z. 161 (1983), 195-210. MF $0498970(58: 16958)$

Institut für Mathematik, Universität Paderborn, D-33095 Paderborn, Germany

E-mail address: kaniuth@math.uni-paderborn.de

Department of Mathematical and Statistical Sciences, University of Alberta, Edmonton, Alberta, Canada T6G 2G1

E-mail address: tlau@math.ualberta.ca

Fakultät für Mathematik, Technische Universität München, D-80290 München, GerMANY

E-mail address: schlicht@ma.tum.de 